\title{
BRAIDED FIELD QUANTIZATION FROM QUANTUM POINCARE COVARIANCE
}

\author{
JERZY LUKIERSKI \\ Institute for Theoretical Physics, University of Wroclaw \\ pl. Maxa Borna 9, 50-206, Wroclaw, Poland \\ lukier@ift.uni.wroc.pl \\ MARIUSZ WORONOWICZ \\ Institute for Theoretical Physics, University of Wroclaw \\ pl. Maxa Borna 9, 50-206, Wroclaw, Poland \\ woronow@ift.uni.wroc.pl \\ Received 27 January 2012 \\ Revised 6 March 2012
}

\begin{abstract}
We demonstrate that the covariance of the algebra of quantum NC fields under quantumdeformed Poincare symmetries implies the appearence of braided algebra of fields and the notion of braided locality in NC QFT. We briefly recall the historical development of NC QFT which was firstly formulated in the framework using classical relativistic symmetries but further it was described as generated by the quantum-deformed symmetries. We argue that consistent covariant quantum-deformed formalism requires "braiding all the way", in particular braided commutator of deformed field oscillators as well as the braid between the field oscillators and noncommutative Fourier exponentials. As example of braided quantum-deformed NC QFT we describe the NC scalar free fields on noncommutative canonical (Moyal-Weyl) space-time with braided $c$-number field commutator which implies braided locality.
\end{abstract}

Keywords: Noncommutative quantum fields; deformed relativistic symmetries; quantum Poincare covariance.

PACS numbers: 11.10.Nx, 02.20 Uw, 02.40 Gh

\section{Introduction}

It is believed that due to quantum gravity (see e.g. Ref. 1) or quantized string effects (see Ref. 2) one should investigate QFT on noncommutative space-times. In such new field-theoretic models the standard Minkowski space-times coordinates $x_{\mu}$ are replaced by the noncommutative ones

$$
\left[x_{\mu}, x_{\nu}\right]=0 \quad \Longrightarrow \quad\left[\hat{x}_{\mu}, \hat{x}_{\nu}\right]=\frac{i}{\kappa^{2}} \Theta_{\mu \nu}(\kappa \hat{x}),
$$


where $\Theta_{\mu \nu}$ is a given tensorial function ${ }^{\mathrm{a}}$

$$
\Theta_{\mu \nu}(\kappa \hat{x})=\Theta_{\mu \nu}^{(0)}+\kappa \Theta_{\mu \nu}^{(1) \rho} \widehat{x}_{\rho}+\cdots,
$$

and $\kappa$ introduced as geometric mass-like parameter. The simplest choice $\Theta_{\mu \nu}(\kappa \widehat{x})=$ $\Theta_{\mu \nu}^{(0)}$ corresponds to the canonical (DFR ${ }^{\mathrm{b} 1}$ or Moyal-Weyl, see e.g. Refs. 4-8) noncommutative space-time and $\Theta_{\mu \nu}(\kappa \widehat{x})=\Theta_{\mu \nu}^{(1) \rho} \widehat{x}_{\rho}$ describes quantum space-time with Lie-algebraic noncommutativity (e.g. $\kappa$-deformed Minkowski ${ }^{9-11}$ ).

Firstly NC free fields were introduced with keeping the classical Poincare symmetry and standard field oscillators algebra unchanged - such approach we call "traditional". The new approach started later, with the introduction of deformed quantum Poincare symmetries as determining the formulation of $\mathrm{NC}$ field theory, with quantum covariance implying definite modification of field oscillators algebra.

1. Traditional approach to NC QFT. Such formalism was firstly elaborated in Refs. 1, 12 for the canonical noncommutative space-time. In such approach it is postulated that the classical Poincare symmetries remain undeformed, with the noncommutativity (1) introducing the breaking of classical Lorentz invariance. The NC quantum free fields $\widehat{\varphi}(\widehat{x})^{\mathrm{c}}$ are obtained by the replacement $x_{\mu} \rightarrow \widehat{x}_{\mu}$ (see (1)) inserted in the standard quantum free scalar KG field $\widehat{\varphi}(x)^{\mathrm{d}}$

$$
\widehat{\varphi}(x)=\frac{1}{(2 \pi)^{4}} \int d^{4} p \delta\left(p^{2}-m^{2}\right) \mathrm{e}^{i p x} a(p),
$$

where the quantized field oscillators satisfy the undeformed (standard) covariant oscillator algebra $\widehat{\mathcal{H}}_{0}$ with the following binary commutation relations

$$
\delta\left(p^{2}-m^{2}\right) \delta\left(q^{2}-m^{2}\right)[a(p), a(q)]=\epsilon\left(p_{0}\right) \delta\left(p^{2}-m^{2}\right) \delta^{(4)}(p+q) .
$$

One can use the Weyl map representing the algebra $\widehat{\mathcal{M}}$ of functions $\widehat{f} \equiv f(\widehat{x})$ on noncommutative space-time (see e.g. Refs. 13, 14)

$$
\widehat{\mathcal{M}}(f(\widehat{x}), \cdot) \stackrel{W}{\simeq} \mathcal{M}(f(x), \star)
$$

with suitable nonlocal $\star$-multiplication. The NC field theory due to the homomorphic Weyl mapping can be represented as a nonlocal theory of standard fields on Minkowski space-time. After the extension of Weyl map (5) to the products of functions depending on different copies of noncommutative Minkowski spaces one

\footnotetext{
${ }^{a}$ We add that one can also consider interesting models with the function $\Theta_{\mu \nu}$ treated as new dynamical "noncommutativity" field (see e.g. Ref. 3).

${ }^{\mathrm{b}} \mathrm{DFR} \equiv$ Dopplicher-Fredenhagen-Roberts

${ }^{\mathrm{c}}$ We denote by $\widehat{\varphi}(x)$ and $\widehat{\varphi}(\widehat{x})$ respectively quantum free fields on standard and noncommutative Minkowski spaces in traditional approach. The standard classical free fields are denoted by $\varphi(x)$; if we substitute $x_{\mu} \rightarrow \widehat{x}_{\mu}$ we obtain the classical NC free fields denoted by $\varphi(\widehat{x})$.

${ }^{\mathrm{d}}$ In this paper for simplicity we shall consider only scalar fields. The standard creation and annihilation operators are defined respectively by $a\left(\vec{p}, p_{0}=\omega(\vec{p})\right)$ and $a\left(\vec{p}, p_{0}=-\omega(\vec{p})\right)$, where $\omega(\vec{p})=\sqrt{\vec{p}^{2}+m^{2}}$ and for real fields $(3) a\left(\vec{p}, p_{0}\right)=a^{\dagger}\left(-\vec{p},-p_{0}\right)$.
} 
can map the algebra of $\mathrm{NC}$ quantum fields into the multilocal algebra of standard quantum fields, in particular ${ }^{\mathrm{e}}$

$$
\widehat{\varphi}(\widehat{x}) \widehat{\varphi}(\widehat{y}) \stackrel{W}{\simeq} \widehat{\varphi}(x) \star \widehat{\varphi}(y) .
$$

Using (6) one can map the commutator of $\mathrm{NC}$ quantum field into the $\star$-commutator of standard quantum fields described in usual Minkowski space by the formula $\left([A, B]_{\star}:=A \star B-B \star A\right)$

$$
[\widehat{\varphi}(\widehat{x}), \widehat{\varphi}(\widehat{y})] \stackrel{W}{\simeq}[\widehat{\varphi}(x), \widehat{\varphi}(y)]_{\star}=i \Delta_{\star}\left(x, y ; m^{2}\right),
$$

where

$$
\begin{gathered}
\Delta_{\star}\left(x, y ; m^{2}\right)=\frac{-i}{(2 \pi)^{3}} \int d^{4} p \delta\left(p^{2}-m^{2}\right) \delta\left(q^{2}-m^{2}\right)\left[a(p) a(q) \mathrm{e}^{i p x} \star \mathrm{e}^{i q y}\right. \\
\left.-a(q) a(p) \mathrm{e}^{i q y} \star \mathrm{e}^{i p x}\right] .
\end{gathered}
$$

Because $\star$-product $\mathrm{e}^{i p x} \star \mathrm{e}^{i p y}$ even in simplest case of canonical noncommutativity (1) is not symmetric, the commutator (4) can not be factored out and the *-commutator (8) is not a $c$-number. In canonical (Moyal-Weyl) case however one can obtain from (8) the Pauli-Jordan relativistic commutator function

$$
\Delta\left(x-y ; m^{2}\right)=\frac{i}{(2 \pi)^{4}} \int d^{4} p \epsilon\left(p_{0}\right) \delta\left(p^{2}-m^{2}\right) \mathrm{e}^{i p x},
$$

if we suitably modify the standard oscillator algebra (4) (see Refs. 7-8).

In traditional NC QFT the standard relativistic locality or microcausality condition can be replaced by the vanishing of $\star-$ commutator for space-like separations of points $x$ and $y\left((x-y)^{2}<0\right)$ (see e.g. Refs. 7, 14), defining $\star$-locality

$$
[\widehat{\varphi}(x), \widehat{\varphi}(y)]=0 \stackrel{N C}{\longrightarrow}[\widehat{\varphi}(x), \widehat{\varphi}(y)]_{\star}=0 .
$$

The traditional approach to NC QFT was further formalized as a modification of Wightmann framework of QFT, with the interpretation of modified locality (10) in terms of so-called wedged geometries (see e.g. Ref. 15).

2. New approach to NC QFT with quantum Poincare symmetries. Such approach firstly appeared with the introduction of canonical noncommutativity relations $\left(\Theta_{\mu \nu}(\kappa \widehat{x})=\Theta_{\mu \nu}^{(0)}\right.$ in $\left.(1)\right)$ as generated by twist factor

$$
\mathcal{F}=e^{\frac{i}{2} \theta^{\mu \nu} P_{\mu} \otimes P_{\nu}}
$$

which determines uniquely the corresponding Hopf-algebraic canonical deformation of Poincare symmetries (Ref. 4, 7, 14). In the framework of twisted Poincare symmetries the wide class of relations (1) are covariant under the action of suitably chosen quantum Poincare algebra generators (see e.g. Ref. 16). If the twist $\mathcal{F}=\mathcal{F}_{(1)} \otimes \mathcal{F}_{(2)}$

${ }^{\mathrm{e}}$ In (6) we describe only the Weyl map for binary field products. 
is known the $\star$-product of $\mathrm{NC}$ quantum fields in new approach is determined by the formulae $e^{4-7}$

$$
\widehat{\phi}(\widehat{x}) \widehat{\phi}(\widehat{y}) \stackrel{W}{\simeq} \widehat{\phi}(x) \star \widehat{\phi}(y) \equiv m\left(\mathcal{F}^{-1} \circ[\widehat{\phi}(x) \otimes \widehat{\phi}(y)]\right)
$$

where we denote by $\widehat{\phi}(x)$ and $\widehat{\phi}(\widehat{x})$ the quantum fields respectively on commutative and noncommutative space-time, which transform under deformed (quantum) Poincare symmetries.

In new approach to NC QFT the algebra of NC fields should be quantumcovariant, i.e. covariant under the Hopf-algebraic action of generators describing quantum relativistic symmetries. In particular it follows that in order to introduce quantum-deformed covariant free field quantization, we should replace the standard field commutator by its quantum-deformed braided version. ${ }^{17-18}$ Further, following several authors (Refs. 7, 14, 19-24), in quantum-covariant formulation of NC quantum fields with quantum Poincare symmetries characterized by the universal $\mathcal{R}$-matrix $\mathcal{R}=\mathcal{R}_{(1)} \otimes \mathcal{R}_{(2)}$, we shall replace the standard commutators in (4) by the following braided commutators defining the field oscillators algebra $\widehat{\mathcal{H}}$

$$
[a(p), a(q)] \quad \longrightarrow \quad[A(p), A(q)]^{B R} \equiv A(p) A(q)-\left(\mathcal{R}_{(2)} \triangleright A(q)\right)\left(\mathcal{R}_{(1)} \triangleright A(p)\right),
$$

where describes the action on the module $\widehat{\mathcal{H}}$ of the deformed Poincare generators.

The aim of this paper is to study the NC quantum fields which are covariant under the quantum-deformed Poincare symmetries, in particular the determination of new algebraic structure of the algebra of such NC quantum free fields $\Phi(\widehat{\phi}(\widehat{x}), \bullet)$ with suitably deformed new $\bullet-$ multiplication. For the products of functions on noncommutative Minkowski space we will employ the Weyl map (5) with its multilocal extension and express the NC quantum fields as nonlocal QFT on classical Minkowski space. Following main ideas of our recent paper ${ }^{25}$ we shall present the formulation of quantum covariant free $\mathrm{NC}$ quantum fields with the introduction of necessary braidings.

In order to introduce the quantum-covariant theory of $\mathrm{NC}$ fields one should use the general covariance properties of tensor product $U \otimes V$ of pair of quantum Poincare algebra modules $U$ and $V$. If the quantum Poincare algebra is characterized by universal matrix $\mathcal{R}=\mathcal{R}_{(1)} \otimes \mathcal{R}_{(2)}$, the transposed tensor product of its modules is given by the following braided transposition ${ }^{f}$ (see e.g. Ref. 26)

$$
\Psi(U \otimes V)=\left(\mathcal{R}_{(2)} \triangleright V\right) \otimes\left(\mathcal{R}_{(1)} \triangleright U\right),
$$

where $\Psi$ is the intertwiner of quantum-deformed Poincare algebra modules. If we choose $U=\widehat{\phi}(\widehat{x}), V=\widehat{\phi}(\widehat{y})$ the quantum-deformed covariant commutator takes the braided form

$$
[\widehat{\phi}(\widehat{x}), \widehat{\phi}(\widehat{y})]_{\bullet}^{B R} \equiv \widehat{\phi}(\widehat{x}) \bullet \widehat{\phi}(\widehat{y})-\left(\mathcal{R}_{(2)} \triangleright \widehat{\phi}(\widehat{y})\right) \bullet\left(\mathcal{R}_{(1)} \triangleright \widehat{\phi}(\widehat{x})\right) .
$$

${ }^{\mathrm{f}}$ Below in Sect. 2 we shall distinguish two different actions of Poincare algebra generators. In formulas (14) and (15) the action $\triangleright$ is still not specified. 
where the action $\triangleright$ on NC fields will be specified later in Sect. 2. The form (15) of braided field commutator with local standard multiplication was proposed firstly by Oeckl, ${ }^{4}$ further used by Zahn ${ }^{17}$ and advocated by Aschieri at all; ${ }^{18}$ one should comment however that the relation (15) is not in common use in NC QFT.

Further we shall use the formula for universal $\mathcal{R}$-matrix describing quantum twist-deformed Poincare symmetries, given by the relations

$$
\mathcal{R}=\mathcal{F}_{21} \mathcal{F}^{-1}=\mathcal{F}^{-2}, \quad \mathcal{F}_{21}=\mathcal{F}_{(2)} \otimes \mathcal{F}_{(1)} .
$$

In order to discuss the braided structure of $\mathrm{NC}$ quantum field theory covariant under quantum Poincare symmetries we shall introduce in Sect. 2 the actions of quantum Poincare algebra generators on the algebra $\Phi(\widehat{\phi}(\widehat{x}), \bullet)$ of quantumdeformed NC fields. We shall define braided covariant product $\widehat{\phi}(\widehat{x}) \bullet \widehat{\phi}(\widehat{y})$ with braid factor (14) describing the exchange of oscillators and noncommutative Fourier exponentials. In Sect. 3 for the case of Moyal-Weyl space-time noncommutativity we shall consider in detail the braided quantization of free scalar quantum NC fields and discuss the braided locality. In last Section in particular we point out that there is alternative way ${ }^{23-24}$ of defining the braiding in the noncommutative quantum field commutators (15) which leads to the triviality of braid factor.

\section{The Covariance of the Algebra of Quantum NC Fields}

In the description of the algebra of quantum NC fields one should answer the following two questions:

- how to define single quantum NC field as describing a representation (module) of quantum Poincare algebra

- how to define the products of quantum NC fields in covariant way, i.e. as a tensorial representation (module) of quantum Poincare algebra (the answer should be given at least for the binary products)

\subsection{Single quantum NC fields as quantum Poincare algebra module}

The quantum NC field $\widehat{\phi}(\widehat{x})$ can be described as infinite sum (in fact continuous integral) of tensor products of noncommutative plane waves $e^{i p \widehat{x}}$ describing the basis of algebra $\widehat{\mathcal{M}}$ and the elements $a(p)$ determining the algebra of field oscillators $\widehat{\mathcal{H}}$

$$
\widehat{\phi}(\widehat{x}) \in \widehat{\mathcal{M}} \underline{\otimes} \widehat{\mathcal{H}},
$$

where $\underline{\otimes}$ denotes braided tensor product with braided $\bullet-$ multiplication (see Sect. 2.2). Using the homomorphic Weyl map of the algebra $\widehat{\mathcal{M}}$ (see (5)) one can represent the noncommutative algebra $\widehat{\mathcal{M}}$ by the algebra $\mathcal{M}$ of classical functions with $\star$-multiplication law. The Weyl map can be applied to the first factor $\widehat{\mathcal{M}}$ in (17) and leads to the $\widehat{\mathcal{H}}$-algebra-valued representation of NC quantum fields, i.e.

$$
\widehat{\phi}(\widehat{x}) \stackrel{W}{\simeq} \widehat{\phi}(x) \in \widehat{\mathcal{H}},
$$


because after the replacement in the Fourier expansion of $\widehat{\phi}(\widehat{x})$ the basis $e^{i p \widehat{x}}$ by $e^{i p x}$ one can use the isomorphism $1 \otimes \widehat{\mathcal{H}} \simeq 1 \otimes \widehat{\mathcal{H}} \simeq \widehat{\mathcal{H}}$. It appears that the algebra of field operators $\widehat{\phi}(x)$ have well defined no-deformation limit $(\widehat{\phi}(x) \rightarrow \widehat{\varphi}(x))$, with the algebra $\widehat{\mathcal{H}}$ becoming the algebra $\widehat{\mathcal{H}}_{0}$ (see (4)).

Let us specify now the action of the deformed Poincare algebra generators on single NC quantum field (17). We shall recall firstly two possible actions of classical Poincare algebra on standard free quantum fields $\widehat{\varphi}(x) \in \widehat{\mathcal{H}}_{0}$ (see $\left.(3),(4)\right)$.

(1) classical differential space-time realization on the functions on classical Minkowski space-time

$$
P_{\mu} \triangleright \widehat{\varphi}(x)=\frac{1}{i} \partial_{\mu} \widehat{\varphi}(x), \quad M_{\mu \nu} \triangleright \widehat{\varphi}(x)=\frac{1}{i} x_{[\mu} \partial_{\nu]} \widehat{\varphi}(x),
$$

(2) quantum-mechanical realization on the free field oscillators algebra

$$
P_{\mu} \triangleright \widehat{\varphi}(x)=\left[P_{\mu}, \widehat{\varphi}(x)\right], \quad M_{\mu \nu} \triangleright \phi=\left[M_{\mu \nu}, \widehat{\varphi}(x)\right],
$$

with $P_{\mu}, M_{\mu \nu} \in \widehat{\mathcal{H}}_{0}$.

The classical Poincare covariance relation

$$
U(\Lambda, a) \widehat{\varphi}(x) U^{-1}(\Lambda, a)=\widehat{\varphi}(\Lambda x+a),
$$

where $U(\Lambda, a)=\exp \left(i a^{\mu} P_{\mu}+i \omega^{\mu \nu} M_{\mu \nu}\right)$ for infinitesimal $a_{\mu}$ and $\omega_{\mu \nu}\left(\Lambda^{\mu}{ }_{\nu}=\delta^{\mu}{ }_{\nu}+\right.$ $\left.\omega^{\mu}{ }_{\nu}\right)$ links two realizations $(19),(20)$. It leads to the following infinitesimal covariance condition

$$
g \triangleright \widehat{\varphi}(x)=-g \triangleright \widehat{\varphi}(x), \quad g=\left(P_{\mu}, M_{\mu \nu}\right) .
$$

We see therefore that for Poincare-covariant standard (undeformed) quantum fields one can use as the action of classical Poincare algebra generators equivalently the "classical" action $\triangleright$ or the quantum-mechanical one $\boldsymbol{}$.

Now we pass to quantum-deformed NC fields (17). At final stage of considerations we shall consider such fields after the Weyl map (18). The realizations (19) and (20) due to deformation are modified, however in covariant theory the covariance condition (22) remains valid provided that we modify the relation (22) as follows ( $S$ is an antipode)

$$
g \triangleright \widehat{\phi}(\widehat{x})=S(g) \triangleright \widehat{\phi}(\widehat{x}) .
$$

By analogy with undeformed case in the tensor product $\widehat{f} \otimes \widehat{h} \in \widehat{\phi}(\widehat{x})(\widehat{f} \in \widehat{\mathcal{M}}$, $\widehat{h} \in \widehat{\mathcal{H}})$ the actions $\triangleright$ on $\widehat{h}$ and $\boldsymbol{\triangleright}$ on $\widehat{f}$ are assumed to be trivial:

$$
g \triangleright \widehat{h}=\epsilon(g) \widehat{h}, \quad g \triangleright \widehat{f}=\epsilon(g) \widehat{f} .
$$

If we use Hopf-algebraic formula (the case of action - is analogous)

$$
g \triangleright(\widehat{f} \otimes \widehat{h})=\Delta(g) \triangleright(\widehat{f} \otimes \widehat{h})=\left(g_{(1)} \triangleright \widehat{f}\right) \otimes\left(g_{(2)} \triangleright \widehat{h}\right),
$$


the actions $g \triangleright \widehat{\phi}(\widehat{x})$ and $g \triangleright \widehat{\phi}(\widehat{x})$ in $(23)$ due to the relations $(24)$ and the structure of coproduct $\Delta(g)$ with unique terms $g \otimes 1$ and $1 \otimes g$ take the form

$$
g \triangleright(\widehat{f} \otimes \widehat{h}) \equiv(g \triangleright \widehat{f}) \otimes \widehat{h}, \quad g \triangleright(\widehat{f} \otimes \widehat{h}) \equiv \widehat{f} \otimes(g \triangleright \widehat{h}) .
$$

After applying the Weyl map (18) one can rewrite (23) in the form similar to $(22)$

$$
g \triangleright \widehat{\phi}(x)=S(g) \triangleright \widehat{\phi}(x),
$$

where commutators in (20) should be replaced by quantum adjoint action

$$
g>\widehat{\varphi}(x)=[g, \widehat{\varphi}(x)] \underset{\text { deformation }}{\stackrel{\text { quantum }}{\Longrightarrow}} g \boldsymbol{\phi}(\widehat{x})=a d_{g} \widehat{\phi}(\widehat{x})=g_{(1)} \widehat{\phi}(\widehat{x}) S\left(g_{(2)}\right) .
$$

If the Hopf-algebraic form of deformed Poincare algebra is known, the formula (28) is fully determined ( $\mathrm{S}$ denotes the antipode); subsequently the action $g \triangleright \widehat{\phi}(x)$ described by the deformation of (19) should be chosen in consistency with the relation $(27)$.

It should be added that one can introduce third possible action $\unrhd$ of generators on NC quantum fields (see e.g. Refs. 23, 24), defined by the formula

$$
g \unrhd(\widehat{f} \otimes \widehat{h})=\left(g_{(1)} \triangleright \widehat{f}\right) \otimes\left(g_{(2)} \triangleright \widehat{h}\right) .
$$

Such action of generators if applied to the field $\widehat{\phi}(\widehat{x})$ due to the relations (26) leads to the following form of covariance conditions (23)

$$
g \unrhd \widehat{\phi}(\widehat{x})=\epsilon(g) \widehat{\phi}(\widehat{x})=0,
$$

which after the Weyl map (18) provides the covariance relation (27).

\subsection{The covariant action of deformed Poincare algebra on the product of NC quantum fields}

In order to formulate the deformed NC QFT we shall define firstly the algebra $\Phi(\widehat{\phi}(\widehat{x}), \bullet)$ of NC fields $\widehat{\phi}(\widehat{x})$ and further perform the Weyl map (see (18)). We multiply the NC fields (17) using the new braided $\bullet-$ multiplication which defines the multiplication of $\mathrm{NC}$ quantum fields with deformed field oscillators algebra

$$
\begin{aligned}
\widehat{\phi}(\widehat{x}) \bullet \widehat{\phi}(\widehat{y}) & =m_{\mathcal{M} \underline{\otimes} \mathcal{H}} \widehat{\phi}(\widehat{x}) \underline{\otimes} \widehat{\phi}(\widehat{y}) \\
& =\left(m_{\mathcal{M}} \otimes m_{\mathcal{H}}\right) \circ\left(i d \otimes \Psi_{\mathcal{M}, \mathcal{H}} \otimes i d\right)[\widehat{\phi}(\widehat{x}) \otimes \widehat{\phi}(\widehat{y})] .
\end{aligned}
$$

Braid factor $\Psi_{\mathcal{M}, \mathcal{H}}$ describes effectively the noncommutativity of factors $A(p)$ and $e^{i q \widehat{y}}$ in the product of field operators $\widehat{\phi}(\widehat{x})$ and $\widehat{\phi}(\widehat{y})$ and it is needed in general case in order to obtain the product $(31)$ as an element of $\widehat{\mathcal{M}}^{(2)} \underline{\otimes \mathcal{H}}$ where by $\widehat{\mathcal{M}}^{(n)}$ we denote the noncommutative functions on the $n$-tuple of quantum Minkowski spaces $\left(\widehat{x}^{(1)}, \widehat{x}^{(2)}, \ldots, \widehat{x}^{(n)}\right)$ (for $n=2$ we have chosen $\widehat{x}^{(1)}=\widehat{x}, \widehat{x}^{(2)}=\widehat{y}$ ). The formula $(31)$ permits to express the basis of binary field products (31)

$$
A=m_{\mathcal{M} \underline{\otimes} \mathcal{H}}\left[\left(e^{i p \widehat{x}} A(p)\right) \underline{\otimes}\left(e^{i q \widehat{y}} A(q)\right)\right]=\left(e^{i p \widehat{x}} A(p)\right) \bullet\left(e^{i q \widehat{y}} A(q)\right),
$$


by the superposition of elements of tensor product $\widehat{\mathcal{M}}^{(2)} \otimes \widehat{\mathcal{H}}$ spanned by the elements

$$
\widetilde{A}=e^{i p \widehat{x}} e^{i q \widehat{y}} \underline{\otimes} A(p) A(q) \in \widehat{\mathcal{M}}^{(2)} \otimes \widehat{\mathcal{H}} .
$$

Further we assume that the quantum deformation of Poincare algebra is described by quasi-triangular Hopf algebra characterized by the universal $\mathcal{R}$-matrix $\mathcal{R}=$ $\mathcal{R}_{(1)} \otimes \mathcal{R}_{(2)}$. Following the general formula (14) we introduce the braid factor expressing the transposition of noncommutative plane waves and deformed field oscillators

$$
\Psi_{\mathcal{M}, \mathcal{H}}\left[A(p) \underline{\otimes} e^{i q \widehat{y}}\right]=\left(\mathcal{R}_{(2)} \triangleright e^{i q \widehat{y}}\right) \underline{\otimes}\left(\mathcal{R}_{(1)}>A(p)\right) .
$$

Using (31) leads to the following equivalent expression for the product (32)

$$
A=\left[e^{i p \widehat{x}} \mathcal{R}_{(2)} \triangleright e^{i q \widehat{y}}\right] \underline{\otimes}\left[\left(\mathcal{R}_{(1)} \triangleright A(p)\right) A(q)\right] .
$$

We recall that we have used here universality of the formula (14) for any two deformed Poincare algebra modules, i.e. the general formula (31) can be rewritten in concrete way as follows

$$
(\widehat{f} \otimes \widehat{h}) \bullet\left(\widehat{f}^{\prime} \otimes \widehat{h}^{\prime}\right)=\left[\widehat{f} \cdot \mathcal{R}_{(2)} \triangleright \widehat{f}^{\prime}\right] \underline{\otimes}\left[\left(\mathcal{R}_{(1)} \triangleright \widehat{h}\right) \cdot \widehat{h}^{\prime}\right] .
$$

where $\widehat{f}, \widehat{f}^{\prime} \in \widehat{\mathcal{M}}$ and $\widehat{h}, \widehat{h}^{\prime} \in \widehat{\mathcal{H}}$. If we perform the Weyl map in the algebra $\widehat{\mathcal{M}}^{(2)}$ $\left(\widehat{f} \stackrel{W}{\rightarrow} f, \widehat{f}^{\prime} \stackrel{W}{\rightarrow} f^{\prime}\right)$ and introduce corresponding star product the first factor in the tensor product on rhs of (36) is becoming a classical function in accordance with the prescription ${ }^{\mathrm{g}}$

$$
\widehat{f} \cdot\left(\mathcal{R}_{(2)} \triangleright \widehat{f}^{\prime}\right) \stackrel{W}{\simeq} f \star\left(\mathcal{R}_{(2)} \triangleright f^{\prime}\right),
$$

and we obtain that

$$
(\widehat{f} \otimes \widehat{h}) \bullet\left(\widehat{f}^{\prime} \otimes \widehat{h}^{\prime}\right) \stackrel{W}{\simeq}\left(f \star\left(\mathcal{R}_{(2)} \triangleright f^{\prime}\right)\left[\left(\mathcal{R}_{(1)} \triangleright \widehat{h}\right) \cdot \widehat{h}^{\prime}\right]\right.
$$

if the relation $\mathbb{C} \otimes \widehat{\mathcal{H}} \simeq \widehat{\mathcal{H}}$ is used.

As we mentioned earlier, we shall use the covariant braided field commutator (15), which after using specified actions $\triangleright, \downarrow$ can be rewritten more explicitly

$$
[\widehat{\phi}(\widehat{x}), \widehat{\phi}(\widehat{y})]^{B R}=\widehat{\phi}(\widehat{x}) \bullet \widehat{\phi}(\widehat{y})-\left[\left(\mathcal{R}_{(2)} \otimes 1\right) \triangleright \widehat{\phi}(\widehat{y})\right] \bullet\left[\left(1 \otimes \mathcal{R}_{(1)}\right) \triangleright \widehat{\phi}(\widehat{x})\right] .
$$

The formula (39) is our basic relation which defines the commutator of free NC quantum fields. We shall show below that the quantum covariance of the product (31) and of deformed commutator requires braid (34) and braided commutator (38)). For covariant NC quantum fields it follows however from (23)

$$
\left(\mathcal{R}_{(2)} \otimes 1\right) \triangleright \widehat{\phi}(\widehat{y})=\left(1 \otimes S\left(\mathcal{R}_{(2)}\right)\right) \triangleright \widehat{\phi}(\widehat{y}),
$$

gFor notational convenience one can introduce the symbol $\circledast$ by means of the formula $(\widehat{f} \otimes \widehat{h}) \bullet$ $\left(\widehat{f}^{\prime} \otimes \widehat{h}^{\prime}\right) \stackrel{W}{\simeq}(f \otimes \widehat{h}) \circledast\left(f^{\prime} \otimes \widehat{h}^{\prime}\right)$. The translation of algebraic properties of $\bullet-$ multiplication $(31)$ (e.g. associativity) into the corresponding properties of $\circledast$ is under consideration. 
i.e. it follows that braided field commutator can be written in other two ways which employs only the action $\triangleright$ or $\boldsymbol{h}^{\mathrm{h}}$

$$
\begin{aligned}
& {[\widehat{\phi}(\widehat{x}), \widehat{\phi}(\widehat{y})]^{B R}=\widehat{\phi}(\widehat{x}) \bullet \widehat{\phi}(\widehat{y})-\mathcal{R}_{21} \triangleright(\widehat{\phi}(\widehat{y}) \bullet \widehat{\phi}(\widehat{x}))} \\
& {[\widehat{\phi}(\widehat{x}), \widehat{\phi}(\widehat{y})]^{B R}=\widehat{\phi}(\widehat{x}) \bullet \widehat{\phi}(\widehat{y})-\mathcal{R}_{21} \bullet(\widehat{\phi}(\widehat{y}) \bullet \widehat{\phi}(\widehat{x})) .}
\end{aligned}
$$

Further we shall employ formula (40) with the quantum-mechanical action $\boldsymbol{~}$. Let us demonstrate firstly that the field product (31) is covariant under the action of a quantum Poincare generator $g \in \mathcal{U}\left(\mathcal{P}_{4}\right)$. Using the Hopf-algebraic formula with fourfold coproduct $\Delta^{(4)}(g)=g_{(1)} \otimes g_{(2)} \otimes g_{(3)} \otimes g_{(4)}$ and the identity $g \triangleright(a \triangleright b)=$ $(g a)>b$ we get

$$
\begin{aligned}
g \triangleright\left[(\widehat{f} \otimes \widehat{h}) \bullet\left(\widehat{f}^{\prime} \otimes \widehat{h}^{\prime}\right)\right] & =\left[\left(g_{(1)}>\widehat{f}\right) \cdot\left(g_{(2)} \mathcal{R}_{(2)}>\widehat{f}^{\prime}\right)\right] \otimes\left[\left(g_{(3)} \mathcal{R}_{(1)}>\widehat{h}\right) \cdot\left(g_{(4)}>\widehat{h}^{\prime}\right)\right] \\
& =\left[\left(g_{(1)}>\widehat{f}\right) \cdot\left(\mathcal{R}_{(2)} g_{(3)}>\widehat{f}^{\prime}\right)\right] \otimes\left[\left(\mathcal{R}_{(1)} g_{(2)}>\widehat{h}\right) \cdot\left(g_{(4)}>\widehat{h}^{\prime}\right)\right] \\
& =\left[\left(g_{(1)}>\widehat{f}\right) \cdot\left(g_{(2)}>\widehat{h}\right)\right] \bullet\left[\left(g_{(3)}>\hat{f}^{\prime}\right) \cdot\left(g_{(4)}>\widehat{h}^{\prime}\right)\right] \\
& =\left[g_{(1)}>(\widehat{f} \cdot \widehat{h})\right] \bullet\left[g_{(2)}>\left(\widehat{f}^{\prime} \cdot \widehat{h}^{\prime}\right)\right]
\end{aligned}
$$

where we use the relations (24) and the equalities ${ }^{\mathrm{i}}$.

$$
g_{(2)}^{I} \mathcal{R}_{(2)}^{J} \otimes g_{(3)}^{I} \mathcal{R}_{(1)}^{J}=\mathcal{R}_{(2)}^{J} g_{(3)}^{I} \otimes \mathcal{R}_{(1)}^{J} g_{(2)}^{I} .
$$

Subsequently, applying (43) to the basis (32) of the product of two NC quantum fields one gets its quantum-Poincare covariance

$$
g \text { \ }(\widehat{\phi}(\widehat{x}) \bullet \widehat{\phi}(\widehat{y}))=m_{\bullet}[\Delta(g) \triangleright \widehat{\phi}(\widehat{x}) \otimes \widehat{\phi}(\widehat{y})]=\left(g_{(1)} \triangleright \widehat{\phi}(\widehat{x})\right) \bullet\left(g_{(2)} \triangleright \widehat{\phi}(\widehat{y})\right) .
$$

Now we shall show the covariance of the braided field commutator. The action of generator $g$ on second term defining braided commutator (39) with action $\boldsymbol{\nabla}$ is

$$
\begin{aligned}
g \triangleright\left(\mathcal{R}_{21} \triangleright[\widehat{\phi}(\widehat{y}) \bullet \widehat{\phi}(\widehat{x})]\right) & =g \triangleright\left[\left(\mathcal{R}_{(2)} \triangleright \widehat{\phi}(\widehat{y})\right) \bullet\left(\mathcal{R}_{(1)} \triangleright \widehat{\phi}(\widehat{x})\right)\right] \\
& =m \bullet\left(\Delta(g) \mathcal{R}_{21} \triangleright \widehat{\phi}(\widehat{y}) \otimes \widehat{\phi}(\widehat{x})\right) .
\end{aligned}
$$

The covariance of braided commutator (39) requires that

$$
g \triangleright \mathcal{R}_{21} \triangleright(\widehat{\phi}(\widehat{y}) \bullet \widehat{\phi}(\widehat{x}))=\mathcal{R}_{21} \triangleright g \triangleright(\widehat{\phi}(\widehat{y}) \bullet \widehat{\phi}(\widehat{x})),
$$

what implies the relation

$$
\Delta(g) \mathcal{R}_{21}-\mathcal{R}_{21} \Delta_{21}(g)=0 .
$$

It is well-known (see e.g Ref. 27) that for any quasitriangular deformed Poincare algebra the relations (48) follow from the definition of universal $\mathcal{R}$-matrix

$$
\Delta_{21}(g)=\mathcal{R} \Delta(g) \mathcal{R}^{-1} .
$$

\footnotetext{
${ }^{\mathrm{h}}$ We use shorthand notation $\mathcal{R}_{21} \triangleright(a \bullet b)=\left(\mathcal{R}_{(2)} \triangleright a\right) \bullet\left(\mathcal{R}_{(1)} \triangleright b\right)$ etc.

${ }^{i}$ See e.g. Ref. 27, Sec . 9.2. We use Sweedler notation with suppressed summation indices. If more explicitly $\Delta^{(4)}(g)=\sum_{I} g_{(1)}^{I} \otimes g_{(2)}^{I} \otimes g_{(3)}^{I} \otimes g_{(4)}^{I}$ and $\mathcal{R}^{(4)}=\sum_{J} 1 \otimes \mathcal{R}_{(1)}^{J} \otimes \mathcal{R}_{(2)}^{J} \otimes 1$ we read e.g. eq. (44) as $\sum_{I} \sum_{J} g_{(2)}^{I} \mathcal{R}_{(2)}^{J} \otimes g_{(3)}^{I} \mathcal{R}_{(1)}^{J}=\sum_{I} \sum_{J} \mathcal{R}_{(2)}^{J} g_{(3)}^{I} \otimes \mathcal{R}_{(1)}^{J} g_{(2)}^{I}$.
} 


\section{Covariant Braided Field Commutator and Braided Field Oscillators Algebra in Twist Deformed QFT}

In twist-deformed quantum field theory the multiplication prescription (31) is determined if we know the twist factor $\mathcal{F}=\mathcal{F}_{(1)} \otimes \mathcal{F}_{(2)}$ and the braid $\Psi_{\mathcal{M}, \mathcal{H}}$ (see (34)). The explicit form of the product of quantum free fields on noncommutative spacetime has therefore a form (we denote $\mathcal{F}^{-1}=\overline{\mathcal{F}}_{(1)} \otimes \overline{\mathcal{F}}_{(2)}$ )

$$
\begin{aligned}
& \widehat{\phi}(\widehat{x}) \bullet \widehat{\phi}(\widehat{y})=m_{\mathcal{M} \underline{\otimes} \mathcal{H}}[\widehat{\phi}(\widehat{x}) \otimes \widehat{\phi}(\widehat{y})] \\
&= \frac{1}{(2 \pi)^{8}} \int d^{4} p \int d^{4} q \delta\left(p^{2}-m^{2}\right) \delta\left(p^{2}-m^{2}\right) \\
& e^{i p \widehat{x}}\left(\mathcal{R}_{(2)} \triangleright e^{i q \widehat{y}}\right) \otimes\left(\mathcal{R}_{(1)} \triangleright A(p)\right) A(q), \\
& \stackrel{W}{\simeq} \frac{1}{(2 \pi)^{8}} \int d^{4} p \int d^{4} q \delta\left(p^{2}-m^{2}\right) \delta\left(p^{2}-m^{2}\right) \\
&\left(\overline{\mathcal{F}}_{(1)} \triangleright e^{i p x}\right)\left(\overline{\mathcal{F}}_{(2)} \mathcal{R}_{(2)} \triangleright e^{i q y}\right)\left(\mathcal{R}_{(1)} \triangleright A(p)\right) A(q),
\end{aligned}
$$

where by the notation $\stackrel{W}{\simeq}$ we denote the Weyl homomorphism in $\widehat{\mathcal{M}}^{(2)}$ with the suitably $\star$-product which represents the product $e^{i p \widehat{x}} e^{i q \widehat{y}}$ in terms of classical Fourier exponentials. The actions of free Poincare generators in $\overline{\mathcal{F}}_{(1)}, \overline{\mathcal{F}}_{(2)}$ on the classical plane waves are described by the differential realization (19) and on the deformed field oscillators the Poincare generators act by the quantum adjoint action (28).

By using (15) and (50) we shall calculate explicitly the braided commutator (42). We get

$$
\begin{aligned}
{[\widehat{\phi}(\widehat{x}), \widehat{\phi}(\widehat{y})]_{\bullet}^{B R}=} & \widehat{\phi}(\widehat{x}) \bullet \widehat{\phi}(\widehat{y})-\mathcal{R}_{21} \triangleright(\widehat{\phi}(\widehat{y}) \bullet \widehat{\phi}(\widehat{x})) \\
\stackrel{\underline{W}}{\underline{(2 \pi)^{8}}} \int d^{4} p \int d^{4} q \delta\left(p^{2}-m^{2}\right) \delta\left(q^{2}-m^{2}\right) & \quad\left[\left(\overline{\mathcal{F}}_{(1)} \triangleright e^{i p x}\right)\left(\overline{\mathcal{F}}_{(2)} \mathcal{R}_{(2)} \triangleright e^{i q y}\right)\left(\mathcal{R}_{(1)} \triangleright A(p)\right) A(q)\right. \\
& \quad-\left(\overline{\mathcal{F}}_{(1)} \triangleright e^{i q y}\right)\left(\overline{\mathcal{F}}_{(2)} \mathcal{R}_{(2)} \triangleright e^{i p x}\right)\left(\mathcal{R}_{(1)} \mathcal{R}_{(2)} \triangleright A(q)\right)\left(\mathcal{R}_{(1)} \triangleright A(p)\right) .
\end{aligned}
$$

Further in order to obtain explicit formulae we shall consider the canonical deformation described by twist (11). As follows from (16) and (11) $\mathcal{R}_{21}$ depends only on the fourmomentum generators $P_{\mu}$ actions given by the formulae

$$
P_{\mu} \triangleright e^{i p x}=p_{\mu} e^{i p x}, \quad P_{\mu} \triangleright A(p)=-p_{\mu} A(p) .
$$

In canonically deformed case we get

$$
\begin{aligned}
\mathcal{R}_{21} \triangleright\left[e^{i p x} \otimes e^{i q y}\right] & =e^{i \theta^{\mu \nu} p_{\mu} q_{\nu}} e^{i p x} \otimes e^{i q y}, \\
\mathcal{R}_{21} \triangleright[A(p) \otimes A(q)] & =e^{i \theta^{\mu \nu} p_{\mu} q_{\nu}} A(p) \otimes A(q),
\end{aligned}
$$

and the braid $\Psi_{\mathcal{M}, \mathcal{H}}$ has the explicit form

$$
\Psi_{\mathcal{M}, \mathcal{H}}\left[A(q) \otimes e^{i p x}\right]=\mathcal{R}_{(2)} \triangleright e^{i p x} \otimes \mathcal{R}_{(1)} \triangleright A(q)=e^{-i \theta^{\mu \nu} p_{\mu} q_{\nu}} e^{i p x} \otimes A(q) .
$$

In order to obtain $c$-number braided field commutator one should be able to factor out in braided field commutator (42) the binary relations satisfied by the 
field oscillators which describe the field oscillators algebra. If we use the formula (34) the required factorization in the braided commutator (42) is achieved by the formula

$$
\begin{gathered}
{[\widehat{\phi}(\widehat{x}), \widehat{\phi}(\widehat{y})]_{\bullet}^{B R} \stackrel{W}{\simeq} \frac{1}{(2 \pi)^{8}} \int d^{4} p \int d^{4} q \delta\left(p^{2}-m^{2}\right) \delta\left(q^{2}-m^{2}\right) e^{i p x} e^{i q y}} \\
{\left[A(p) \star \mathcal{H} A(q)-\mathcal{R}_{21} \triangleright(A(q) \star \mathcal{H} A(p))\right],}
\end{gathered}
$$

where the new multiplication describing the binary oscillator algebra relation is the following

$$
A(p) \star_{\mathcal{H}} A(q)=m \circ \mathcal{F} \triangleright[A(p) \otimes A(q)]=e^{\frac{i}{2} \theta^{\mu \nu} p_{\mu} q_{\nu}} A(p) A(q) .
$$

We point out here that the multiplication $\star_{\mathcal{H}}$ is an inverse of the $\star$-multiplication $\left(\mathcal{F}^{-1}\right.$ in $\left.(12)\right)$ is replaced in $(56)$ by $\left.\mathcal{F}\right)$ but it is known from the literature (e.g. it was used in Ref. 7) as describing deformed oscillators algebra.

The following modification of standard free field oscillators algebra (4) describes the binary relation for deformed field oscillators

$\delta\left(p^{2}-m^{2}\right) \delta\left(q^{2}-m^{2}\right)\left[A(p) \star_{\mathcal{H}} A(q)-\mathcal{R}_{21} \triangleright\left(A(q) \star_{\mathcal{H}} A(p)\right)\right]=\epsilon\left(p_{0}\right) \delta\left(p^{2}-m^{2}\right) \delta^{(4)}(p+q)$.

The choice (57) leads to desired properties, namely:

(1) due to the presence of braid factor $\mathcal{R}_{21}$ the deformed oscillators algebra is covariant under quantum Poincare symmetries

(2) it leads to $c$-number value of the braided commutator (55)

If we substitute (57) into (55) we obtain the final formula

$$
[\widehat{\phi}(\widehat{x}), \widehat{\phi}(\widehat{y})]_{\bullet}^{B R} \stackrel{W}{\simeq} \Delta\left(x-y ; m^{2}\right),
$$

with the braided commutator for canonically deformed free quantum fields given by known standard Pauli-Jordan function (see (9)) .

It should be noted that the choices of $\star$-multiplication (see (12)) and of the covariant braid $\Psi_{\mathcal{M}, \mathcal{H}}$ (see (34) are necessary for getting the twist-covariant algebra of deformed field operators. The braid factor $\mathcal{R}_{21}$ which is an intertwiner in quantum quasitriangular Poincare-Hopf algebra appears in our framework on three levels:

(1) in the Weyl realization of the algebra $\widehat{\mathcal{M}}$ as expressing the "braided commutativity" of the algebra of classical fields on Minkowski space-time with the $\star$-multiplication (see e.g. Ref. 18).

(2) in the algebra $\widehat{\mathcal{H}}$ (see $(57)$ ) of quantized field oscillators which is covariant under the action $>$ of the Poincare symmetry generators $g$.

(3) in the algebra $\Phi(\widehat{\phi}(\widehat{x}), \bullet)$ of deformed NC free quantum field $\widehat{\phi}(\widehat{x})$ (see $(17))$ firstly in the definition of multiplication $m_{\mathcal{M} \otimes \mathcal{H}}$ (see (31)), and further in the definition of braided field commutator (42). 
We point out that the nonstandard multiplication of the deformed oscillators given by (56) and the relation (57) are selected by the requirement of braided --locality of NC quantum free fields or equivalently by the $c$-number value of braided field commutator (see (58)). In NC QFT covariant under quantum Poincare symmetries the standard locality condition is therefore modifed not only by the introduction of $\star-$ multiplication of fields on Minkowski space but also by the deformation of field commutator into the braided one. Let us observe that in the example of canonical twist (11) the rhs of (51) and (55) describing the braided commutator of fields $\widehat{\phi}(x), \widehat{\phi}(y)$ after the Weyl map vanishes if the points $x, y$ are separated in space-like way $\left((x-y)^{2}<0\right)$. We see that the modification of locality in traditional approach (see $(10))$ is replaced by the following braided $\bullet$-locality relation which should be understood after performing the Weyl map which introduces classical space-time points $x, y^{\mathrm{j}}$

$$
[\widehat{\varphi}(x), \widehat{\varphi}(y)]=0 \underset{\text { deformation }}{\stackrel{\text { quantum }}{\Rightarrow}}[\widehat{\phi}(\widehat{x}), \widehat{\phi}(\widehat{y})]_{\bullet}^{B R} \stackrel{W}{\simeq} 0 .
$$

The formula (58) provides an explicit example of NC quantum field satisfying the braided $\bullet$-locality condition (59).

\section{Conclusions}

In this paper we present the quantum-covariant braided formulation of the theory of noncommutative quantum free fields. We restricted our considerations to binary products of such fields. For twist-deformed noncommutative fields the extension of our formalism to n-ary associative products is rather straightforward, with the associativity of braided field products following from the hexagon relation satisfied by braid $\mathcal{R}_{21}$ (see e.g. Ref. 27 ).

From our quantum covariance requirements we obtained the braided form (15) of deformed field commutator, the braided form (13) of deformed oscillator algebra and braided •-multiplication (31) in the algebra of deformed quantum free fields. We add that the elements of our construction were present in previous papers. ${ }^{7,17,23-24}$ The closest to our consideration is the approach of Fiore ${ }^{23-24}$ where the braided commutator is however defined with braid factor $\mathcal{R}_{21}$ acting on fields $\widehat{\phi}(\widehat{x})$ by the action $\unrhd$, described by (29) (this was already implicit in the second option of Ref. 7 , see formula (46) there). In such a case the braid factor becomes trivial, because due to the relations $\varepsilon\left(\mathcal{R}_{(1)}\right) \otimes \mathcal{R}_{(2)}=\mathcal{R}_{(1)} \otimes \varepsilon\left(\mathcal{R}_{(2)}\right)=\mathbf{1} \otimes \mathbf{1}(\varepsilon$ is the counit) and (30) we get $\mathcal{R}_{(1)} \unrhd \widehat{\phi}(\widehat{x}) \otimes \mathcal{R}_{(2)}=\widehat{\phi}(\widehat{x}) \otimes \mathbf{1}, \mathcal{R}_{(2)} \unrhd \widehat{\phi}(\widehat{x}) \otimes \mathcal{R}_{(1)}=\widehat{\phi}(\widehat{x}) \otimes \mathbf{1}$, whence

$$
g \unrhd(\widehat{\phi}(\widehat{y}) \widehat{\phi}(\widehat{x}))=\widehat{\phi}(\widehat{y}) \widehat{\phi}(\widehat{x}),
$$

and the braided field commutator becomes a standard one. The latter also gives the c-number function at the rhs of (58), which fulfills (59) at spacelike distances.

jIf we introduce the extension of the $\star$-product on $\mathcal{M}$ into the symbol $\circledast$ (see footnote g), the braided $\star$-locality can be rewritten as $[\widehat{\phi}(\widehat{x}), \widehat{\phi}(\widehat{y})]_{\circledast}=0$ for $(x-y)^{2}<0$ where $[A, B]_{\circledast}=A \circledast B-B \circledast A$. 
Our explicit calculations have been given for the simplest case of canonical twist deformation. If however the twist factor depends as well on the Lorentz generators $M_{\mu \nu}$ (see e.g. Ref. 16), the formulae describing the algebra of deformed quantum fields are more complicated. In such a case after the Weyl map the bidifferential operator describing $\star$-product in algebra $\widehat{\mathcal{M}}$ depends also on the space-time coordinate $x_{\mu}$ and explicit calculations are much more complicated. However, in principle the presented here braided fields approach can be applied to general quasitriangular quantum deformation of free quantum fields.

\section{Acknowledgments}

We would like to thank Gaetano Fiore for reading the paper and providing important comments. JL is thankfull to Goran Djordjevic and Jelena Stankovic for the invitation to participate in Memorial Julius Wess 2011 Workshop. The paper was supported by Polish NCN grant 2011/01/B/ST2/03354.

\section{References}

1. S. Doplicher, K. Fregenhagen and J. E. Roberts, Phys. Lett. B 331, 39 (1994); Comm. Math. Phys. 172, 187 (1995).

2. N. Seiberg and E. Witten, JHEP 9909, 032 (1999).

3. R. Amorim, Phys. Rev. Lett. 102, 081602 (2008).

4. R. Oeckl, Nucl. Phys. B 581, 559 (2000).

5. M. Chaichian, P. P. Kulish, K. Nishijma, A. Tureanu, Phys. Lett. B 604, 98 (2004).

6. J. Wess, Deformed Coordinate Spaces Derivatives, in Proc. BW2003 Workshop Mathematical, Theoretical and Phenomenological Challenges beyond the Standard Model - Perspectives of Balkan Collaborations, eds. G. Djordjevic, Lj. Nesic and J. Wess (World Scientific, Singapore, 2005), p. 122.

7. G. Fiore and J. Wess, Phys. Rev. D 75, 105022 (2007).

8. A. P. Balachandran, A. Pinzul and B. A. Qureshi, Phys. Rev. D 77, 025021 (2008).

9. S. Zakrzewski, Journ. of Phys. A 27, 2079 (1994).

10. S. Majid and H. Ruegg, Phys. Lett. Phys. Lett. B 329, 189 (1994).

11. J. Lukierski, H. Ruegg and W. J. Zakrzewski, Annals of Phys. 243, 90 (1995).

12. T. Filk, Phys. Lett. B 376, 53 (1996).

13. C. Blohman, J. Math. Phys. 44, 4736 (2003).

14. P. P. Kulish, in Proc. Karlstad Conf. (July 2004), Satelite Conf. of IV-th European Congress of Math., ed. AMS, Contemp. Math. 391, 213 (2005).

15. H. Grosse and G. Lechner, JHEP 0809, 131 (2008); JHEP 0711, 012 (2007).

16. J. Lukierski and M. Woronowicz, Phys. Lett. B 633, 116 (2006).

17. J. Zahn, Phys. Rev. D 73, 105005 (2006).

18. P. Aschieri, F. Lizzi and P. Vitale, Phys. Rev. D 77, 025037 (2008).

19. M. Daszkiewicz, J. Lukierski and M. Woronowicz, Mod. Phys. Lett. A 23, 9 (2008).

20. M. Daszkiewicz, J. Lukierski and M. Woronowicz, Phys. Rev. D 77, 105007 (2008).

21. C. A. S. Young and R. Zegers, Nucl. Phys. B 797, 537 (2008).

22. T. R. Govindarajan, K. S. Gupta, E. Harikumar, S. Meljanac and D. Meljanac, Phys. Rev. D 80, 025014 (2010). 
23. G. Fiore, Quantum Field Theory and Beyond, in Proc. Symposium in honor of Wolfhart Zimmermann's 80th birthday, (Ringberg Castle 02/2008). eds. E. Seiler and K. Sibold, (World Scientific Publi. Co., 2008), pp. 64-84.

24. G. Fiore, Jour. Phys. A 43, 155401 (2010).

25. J. Lukierski and M. Woronowicz, arXiv:1105.3612 [hep-th].

26. S. Majid, Mod. Phys. A 5, 1 (1990).

27. S. Majid, Foundations of Quantum Group Theory, Sect. 9, (Cambridge Univ. Press 1996). 